\title{
Stem cells of the endometrium: a leap towards regenerative medicine
}

\begin{abstract}
Stem cells derived from umbilical cord, peripheral blood, bone marrow and adipose tissue are routinely been used to treat disease today. Nevertheless, the existence of adult stem cells and their immense potency from different sources such as placenta, endometrium, fallopian tube, amniotic fluid, amniotic membrane, menstrual blood have been discovered in the past decades. Stem cells derived from female reproductive sources, especially endometrium are gaining special interest in recent years. It is a dynamic tissue engaged in the coordinated functions of proliferation, differentiation and menstrual shedding. Upper functional is layer which sheds during menstruation is later regenerated efficiently by the basal is layer of the endometrial tissue, suggesting the presence of highly potential stem cell population. In addition, endometrium can easily be obtained from hysterectomy (surgical discards of uterus), thus making it easier to collect. Besides, they are reliable, accessible, non-invasive and ideal alternate potent source of stem cells for therapeutic interventions. Thus, the aim of this present review is to highlight the significance of these stem cells isolated from endometrium for their implications in regenerative stem cell therapeutics.
\end{abstract}

Volume 4 Issue 6- 2017

\author{
Apurva Birajdar,' Rakesh Sharma, ${ }^{2}$ Priyanka \\ Hilage,' Sangeeta Desai, ${ }^{3}$ Indumathi \\ Somasundaram ' \\ 'Department of Stem cell and Regenerative Medicine, D.Y. Pati \\ University, India \\ ${ }^{2}$ Dept of Obstetrics and Gynaecology, D.Y. Patil Medical College \\ and University, India
}

\author{
Correspondence: Indumathi Somasundaram, Department \\ of Stem cell and Regenerative Medicine, Centre for \\ Interdisciplinary research, D.Y. Patil University, Kolhapur, \\ Maharashtra, India, Tel +91-8520802540, \\ Email industemcell0I@gmail.com
}

Received: February II, 2017 | Published: April 06, 2017

Keywords: endometrium, menstrual blood, stem cells, regenerative medicine, endometrial dysfunctions

\section{Introduction}

Stem cells in recent years have configured regenerative medicine with their wide range of therapeutic potentials. This revolutionary change has increased the demand of stem cells for myriad of diseases. Their ability to treat many diseases rests on their unique properties of self renewal and differentiation. Stem cells and its therapeutic implications from maternal sources such as Placenta, ${ }^{1}$ amniotic fluid, ${ }^{2,3}$ amniotic membrane,${ }^{4}$ menstrual blood, ${ }^{5}$ endometrial tissue, ${ }^{6-8}$ fallopian tube, ${ }^{9}$ and even breast milk, ${ }^{8,10}$ are underway and has been a fascinating area of research in recent years. Research on maternal sources of stem cells is most important to target the major health challenges facing women and children around the world. Despite the existence of other maternal sources, uterus being the largest female reproductive organ gains much attention as a potential source for stem cells. Based on the dynamic tissue remodelling in all compartments of the uterus, during the menstrual cycle and pregnancy, it has been suggested that adult stem cells of the endometrium must play a role in uterine tissue maintenance and function and marked by its high self renewal ad regenerative potential. ${ }^{6,11}$ In addition, it has been postulated that abnormalities of these stem cells forms the basis for a wide range of gynaecological disorders. ${ }^{12}$ Thus, it is essential to identify the inherent property of these stem cells for regenerative medicine and determine their possible roles in causing gynaecologic diseases, thereby considering them as a possible therapeutic target for curative therapeutics.

\section{Endometrial stem cells}

Human endometrium lines the uterine cavity as far the isthmus of the uterus, where it becomes continuous with the lining of the cervical canal. The endometrium begins to reach full development at puberty and thereafter exhibits dramatic changes during each menstrual cycle. ${ }^{13}$ It undergoes further changes before, during, and after pregnancy, during the menopause, and in old age. The endometrium is a simple columnar epithelium. It is divided into two zones, the inner functionalis which is adjacent to the uterine cavity and a deeper basalis layer which overlies the myometrium. The functionalis layer is shed each month with menstruation, and is then regenerated from the basalis layer which is not shed. The lower basalis contains the basal region of the uterine glands, dense stroma (that remains relatively unaltered during the menstrual cycle), large blood vessel remains and lymphoid aggregates. It serves as the germinal compartment for generating new functionalis each month. ${ }^{14}$

It has been postulated that the niche of these adult stem or progenitor cells of the endometrium is the lower basalis. These stem or progenitor cells were also identified to be in the trophic endometrium of post menopausal women. ${ }^{15}$ Accumulating evidences report on the existence of epithelial and stromal stem cells in endometrial tissue. ${ }^{15-18}$ Studies have substantiated the remarkable capacity of endometrial regeneration. Each menstrual cycle is associated with the vascular proliferation, glandular secretion and the endometrial growth. Absence of progesterone, the demise of corpus luteum and the subsequent fall in circulating progesterone lead to vasoconstriction, necrosis of the endometrium and menstruation. For these reasons, reliable studies on menstrual blood derived stem cells are in process and reported to provide great promises for use in tissue repair and treatment of diseases, due to the plasticity and longevity of the cells. Although menstrual blood has proven to be a unique and novel source of stromal cells from the endometrial functionalis, putative adult stem or progenitor cells that are responsible for the cyclical regeneration of the endometrium functionalis, every month reside in the basalis region of the endometrium, as described earlier. Based on the dynamic tissue remodelling in all compartments of the uterus, during the menstrual cycle and pregnancy, it has been suggested that adult stem 
cells from the endometrial tissue plays a vital role. Hence, a thorough characterization of the uterine/endometrial stem cells derived from the endometrial tissue biopsy of the inner lining of the uterus is underway.

\section{Advantages of endometrial stem cells}

Bone marrow acts as a conventional and most ideal source of stem cell transplantation in regenerative medicine since many decades. However, its disadvantages, such as invasive procedure, low yield, age-related decline in its potency, anesthesia and so on limits the applicability. ${ }^{19}$ Endometrial stromal cells possess wide range of advantages as opposed to other post-natal stem cells, including bone marrow to prove them as a valuable tool in cell-based therapies. They are as follows: easy to isolate, readily available, non- invasive, high accessibility, trash source, longer preservation, highly clonogenic with a higher multi-differentiation ability, ${ }^{15,20}$ possess immunomodulatory properties $^{21}$ and inherent angiogenic potential. ${ }^{22}$ Besides, angiogenesis starts in-utero and the regenerative potency could easily be restored with estrogen treatment even after menopause, proving their high regenerative efficacy. Additionally there are several hysterectomies performed yearly all over the world that could serve as a donor bank providing stem cells for both men and women in treating diseases. Besides, the endometrium provides a source of immunologically matched cells for tissue engineering without concern for rejection. These dynamic properties of endometrial stem cells with their inherent potency makes them ideal source for their regenerative applications.

\section{Applications of endometrial stem cells}

Several in-vitro studies on isolation, culture and characterization of epithelial and stromal cells from endometrium are reported. Recently, endometrial cells were also supposed to be resided in a perivascular niche of the endometrium..$^{21,23}$ From our study, we found that endometrial stem cell possess remarkable characteristic marker profile panel including CD146, CD140b pervivascular population and other mesenchymal markers such as CD90, CD73, CD105, CD44, CD29, CD166, CD54 etc. ${ }^{18}$ Besides, their high angiogenic potency was also reported, proving their efficacy in treating vascular diseases. This is evidenced by several pre-clinical and clinical trials on endometrial stem cells in autologous/allogenic transplantations. Endometrial stem cells have already entered pre-clinical and clinical trial in treating various disorders including cardiovascular diseases, ${ }^{24,25}$ Parkinson's disease ${ }^{26}$ diabetes ${ }^{27}$ and stroke. ${ }^{28}$

The first report of clinical use of endometrial regenerative cells isolated form menstrual blood (ERC) involved treating patients with multiple sclerosis. ${ }^{29}$ No adverse events were reported at the time of last follow-up. An experimental study demonstrated that menstrual blood-derived stromal cells promoted functional improvement of damaged heart tissue. ${ }^{24} \mathrm{He}$ also demonstrated the therapeutic efficacy of both MenScs and EnScs in vivo and in vitro. Borlongan et al., ${ }^{28}$ demonstrated embryonic-like stem cell phenotypic markers, such as Oct4, SSEA, and Nanog, and other neural markers upon its culture. ${ }^{28}$ Wolff et al., ${ }^{26}$ reported the use of in-vitro endometrial-derived neural cells in a Parkinson's disease immunocompetent mouse model using sample of nine reproductively active women. ${ }^{26}$ These cells restored dopamine levels in mice upon transplantation. The study conducted by Hugh Taylor demonstrated the potential of endometrial stem cells for therapeutic purpose in patients with type I diabetes. ${ }^{27}$ Following this, several other studies are underway, and endometrial stem cells have a proven record of being a valuable ideal source of stem cell therapeutics. Thus, it is evident that endometrial stem cells possess ability to treat human diseases. There are much more to be explored in endometrial stem cells, to prove themselves as an ideal candidate for regenerative therapeutic applications.

\section{Challenges and future scope}

Endometrial stem cell, in the near future, will become an ultimate source of regenerative medicine in treating all possible diseases, especially very sooner in the field of vascular biology applications, due to their dynamicity and innate angiogenic potency. Although, endometrial stem cells have convincing track record to call themselves as an ideal candidate in regenerative medicine, it possesses a threat by causing several gynecological disorders. Endometrial eptithelial/ stromal population are the culprit in the pathophysiology of diseases such as endometriosis, endometrial hyperplasia, endometrial cancer, and adenomyosis through their abnormal proliferation, differentiation, alterations in the function, regulation, and location. Thus, besides studying the regenerative aspects of endometrial stem cells, in-depth understanding of the other side of the coin, the role of these stem cells in causing these gynecological diseases may unravel several unresolved barriers of uterine disorders and pave way for breakthrough studies of targeted therapeutic approach of targeting these uterine diseases by serving the woman kind.

\section{Acknowledgements}

None.

\section{Conflict of interest}

The author declares no conflict of interest.

\section{References}

1. Sabapathy V, Ravi S, Srivastava V, et al. Long-term cultured human term placenta-derived mesenchymal stem cells of maternal origin displays plasticity. Stem Cells Int. 2012;2012:174-328.

2. Kim J, Lee Y, Kim H, et al. Human amniotic fluid-derived stem cells have characteristics of multipotent stem cells. Cell Prolif. 2007;40(1):75-90.

3. In 't Anker PS, Scherjon SA, Kleijburg-van der Keur C, et al. Amniotic fluid as a novel source of mesenchymal stem cells for therapeutic transplantation. Blood. 2003;102(4):1548-1549.

4. Warrior S, Haridas N, Bhonde R. Inherent propensity of amnion-derived mesenchymal stem cells towards endothelial lineage: vascularisation from an avascular tissue. Placenta. 2012;33(10):850-858.

5. Musina RA, Belyavski AV, Tarusova OV, et al. Endometrial mesenchymal stem cells isolated from the menstrual blood. Bull Exp Biol Med. 2008;145(4):539-543.

6. Gargett CE, Schwab KE, Zillwood RM, et al. Isolation and culture of epithelial progenitors and mesenchymal stem cells from human endometrium. Biol Reprod. 2009;80(6):1136-1145.

7. Schwab KE, Hutchinson P, Gargett CE. Identification of surface markers for prospective isolation of human endometrial stromal colony forming cells. Hum Reprod. 2008;23(4):934-943.

8. Indumathi S, Dhanasekaran M, Rajkumar JS, et al. Exploring the stem cell ad non-stem cell constituents of human breast milk. Cytotechnology. 2013;65(3):385-393.

9. Jazedje T, Perin PM, Czeresnia CE, et al. Human fallopian tube: a new source of multipotent adult mesenchymal stem cells discarded in surgical procedures. J Transl Med. 2009;7:46. 
10. Kaingade PM, Somasundaram I, Nikam AB, et al. Assessment of growth factors secreted by human breast milk mesenchymal stem cells. Breastfeed Med. 2015;11(1):26-31.

11. Dimitrov R, Timeva T, Kyurkchiev D, et al. Characterization of clonogenic stromal cells isolated from human endometrium. Reproduction. 2008;135(4):551-558.

12. Figueira PG, Abrão MS, Krikun G, et al. Stem cells in endometrium and their role in the pathogenesis of endometriosis. Ann N Y Acad Sci. 2011;1221:10-17.

13. Brosens JJ, Parker MG, McIndoe A, et al. A role for menstruation in preconditioning the uterus for successful pregnancy. Am J Obstet Gynecol. 2009;200(6):611-616.

14. Spencer TE, Hayashi K, Hu J, et al. Comparative Developmental Biology of the mammalian uterus. Curr Top Dev Biol. 2005;68:85-122.

15. Gargett CE, Chan RW, Schwab KE. Endometrial stem cells. Reproductive endocrinology. 2007;19(4):377-383.

16. Figueira PG, Abrão MS, Krikun G, et al. Stem cells in endometrium and pathogenesis of endometrium. Ann Y N Acad Sci. 2011;1221(1):10-17.

17. Cho NH, Park YK, Kim YT, et al. Lifetime expression of stem cell markers in the uterine endometrium. Fertile Steril. 2004;81(2):403-407.

18. Indumathi S, Harikrishnan R, Rajkumar JS, et al. Prospective biomarkers of stem cells of human endometrium and fallopian tube in comparison to bone marrow. Cell Tissue Res. 2013;352(3):537-549.

19. Rebelatto CK, Aguiar AM, Moretão MP, et al. Dissimilar Differentiation of Mesenchymal Stem Cells from Bone Marrow, Umbilical Cord Blood, and Adipose Tissue. Exp Biol Med (Maywood). 2008;233(7):901-913.

20. Gargett CE, Masuda H. Adult stem cells in the endometrium. Mol Hum Reprod. 2010;16(11):818-834.
21. Spitzer TL, Rojas A, Zelenko Z, et al. Perivascular human endometrial mesenchymal stem cells express pathways relevant to self-renewal, lineage specification, and functional phenotype. Biol Reprod. 2012;86(2):58.

22. Gargett CE, Rogers PA. Human endometrial angiogenesis. Reproduction. 2001;121(2):181-186

23. Schwab KE, Gargett CE. Co-expression of two perivascular cell markers isolates mesenchymal stem-like cells from human endometrium. Hum Reprod. 2007;22(9):2903-2911.

24. Hida N, Nishiyama N, Miyoshi S, et al. Novel cardiac precursor- like cells from human menstrual blood-derived mesenchymalcells. Stem Cells. 2008;26(7):1695-1704.

25. Ikegami Y, Miyoshi S, Nishiyama N, et al. Serum-independent cardiomyogenic transdifferentiation in human endometrium-derived mesenchymal cells. Artif Organs. 2010;34(4):280-288.

26. Wolff EF, Gao XB, Yao KV, et al. Endometrial stem cell transplantation restores dopamine production in a Parkinson's disease model. J Cell Mol Med. 2011;15(4):747-755.

27. Santamaria X, Massasa EE, Feng Y, et al. Derivation of insulin producing cells from human endometrial stromal stem cells and use in the treatmen of murine diabetes. Mol Ther. 2011;19(11):2065-2071.

28. Borlongan CV, Kaneko Y, Maki M, et al. Menstrual blood cells display stem cell-like phenotypic markers and exert neuroprotection following transplantation in experimental stroke. Stem Cells Dev. 2010;19(4):439452.

29. Zhong Z, Patel AN, Ichim TE, et al. Feasibility investigation of allogeneic endometrial regenerative cells. J Transl Med. 2009;7:15. 